\title{
Targeting the accomplice to thwart the culprit: a new target for the prevention of amyloid deposition
}

\author{
David R. Borchelt \\ Department of Neuroscience, Center for Translational Research in Neurodegenerative Disease, McKnight Brain Institute, University of Florida, Gainesville, Florida, USA.
}

\begin{abstract}
Inheritance of the E4 allele of the apolipoprotein E gene (APOE4) substantially increases the risk of developing late-onset Alzheimer disease (AD). A large body of evidence has firmly established a role for apoE in modulating the risk of developing the amyloid plaque pathology that is pathognomonic for $A D$. In this issue of the $J C l$, Liao and colleagues discovered that antibodies against a nonlipidated form of apoE4 are highly effective in delaying the deposition of amyloid $\beta(A \beta)$ peptides in mouse models of $A D$ pathology. Using a combination of passive immunization and viral-mediated expression of recombinant antibodies, the authors show that Fc receptor-mediated clearance of the nonlipidated apoE4 was critical in delaying $A \beta$ deposition. Collectively, this study identifies a new therapeutic target that could be exploited to prevent, or possibly reverse, the $A \beta$ pathology of $A D$.
\end{abstract}

\section{Introduction}

One of the most replicated genetic association studies in Alzheimer disease (AD) is the discovery that the apolipoprotein $\mathrm{E}$ (APOE) genotype modulates the risk of developing $\operatorname{AD}(1,2)$. Notably, apoE immunoreactivity was first described in association with both amyloid $\beta(\mathrm{A} \beta)$ plaques and neurofibrillary tangles in humans well before genetic studies linked APOE to AD (3). The major alleles of human $A P O E$ are $E 2, E 3$, and $E 4$, all producing 299 aasecreted products that differ at aa 112 and 158 as follows: E2, Cys/Cys; E3, Cys/Arg; $E 4, \mathrm{Arg} / \mathrm{Arg}$ (reviewed in ref. 4). E2 is the least common and E3 is the most common allele. Forty percent of all patients with sporadic AD have 1 allele of APOE4, and the risk increases 5- to 10-fold in subjects with 2 alleles (5). APOE has been implicated in a plethora of pathways that could potentially modulate the risk for $\mathrm{AD}$, including modulating the toxicity of tau pathology (6), modulating neuroinflammation, impairing mitochondrial function, and altering lipid metabolism (reviewed in ref. 7). However, studies in humans and preclinical mouse models have firmly established that the presence of APOE4 leads to earlier onset of amyloid pathology (recently reviewed in ref. 8), suggesting that the primary mechanism by which apoE modulates the risk of AD is by modu-

\section{Antibodies against nonlipidated apoE emerge as new biotherapies for AD prevention}

The study by Liao and colleagues evaluated a series of antibodies against human apoE (9). Through careful analysis they determined that antibodies against a nonlipidated, possibly aggregated, form of apoE4 are highly effective in delaying the deposition of $\mathrm{A} \beta$ peptides in mice that express human mutant amyloid precursor protein (APP), human mutant presenilin (PS1), and human apoE4. Beginning with passive immunization to screen antibodies, the antibody that emerged as most efficacious was poorly reactive to lating the deposition of $A \beta$.

Related Article: p. 2144

Conflict of interest: The author has declared that no conflict of interest exists.

Reference information: / Clin Invest. 2018;128(5):1734-1736. https://doi.org/10.1172/JCI120414

lipidated forms of apoE3 or apoE4. Using adeno-associated virus-mediated CNS expression of recombinant antibodies, the authors show that Fc receptor-mediated clearance of the nonlipidated apoE4 was critical in delaying $A \beta$ deposition. Passive immunization with antibodies that recognized the lipidated forms of apoE was ineffective, due partially to binding to lipidated apoE in plasma and rapid clearance of the immune complex. The antibodies to nonlipidated apoE4 were highly reactive to cored-neuritic plaques in the APP/PS1 mice, suggesting that the nonlipidated protein closely associates with $A \beta$ aggregates. Thus, the presumptive mechanism by which these antibodies delay the deposition of $\mathrm{A} \beta$ is through microglial-mediated phagocytosis of APOE $4 / \mathrm{A} \beta$ complexes that form early in the formation of $A \beta$ deposits (Figure 1). Whether these antibodies could be effective in promoting the clearance of preexisting $A \beta$ deposits requires further investigation.

Whether nonlipidated apoE is an active or passive accomplice in the deposition of $A \beta$ is unclear. Studies in transgenic mice have generally shown that apoE plays a pivotal role in $A \beta$ deposition. Targeted inactivation of the endogenous apoE allele in mice that overexpress mutant APP profoundly inhibits $A \beta$ deposition (10). Targeted replacement of endogenous apoE alleles with human $A P O E$ alleles has demonstrated that the APOE4 allele is significantly more amyloidogenic than the $E 2$ or $E 3$ alleles $(11,12)$. More recently, a pair of studies demonstrated that apoE is critical in the early stages of $A \beta$ oligomerization and assembly, showing much less influence once deposition has taken hold (13, 14). Most effort in the field has focused on the lipidated forms of apoE. Studies have shown that lipidated apoE4 preferentially stabilizes $A \beta$ oligomers (15), selectively promotes $A \beta$ fibrillization $(16,17)$, and has a greater affinity for $A \beta$ peptides (2, 18). Early studies with recombinant apoE isolated from $E$. coli reported that nonlipi- 


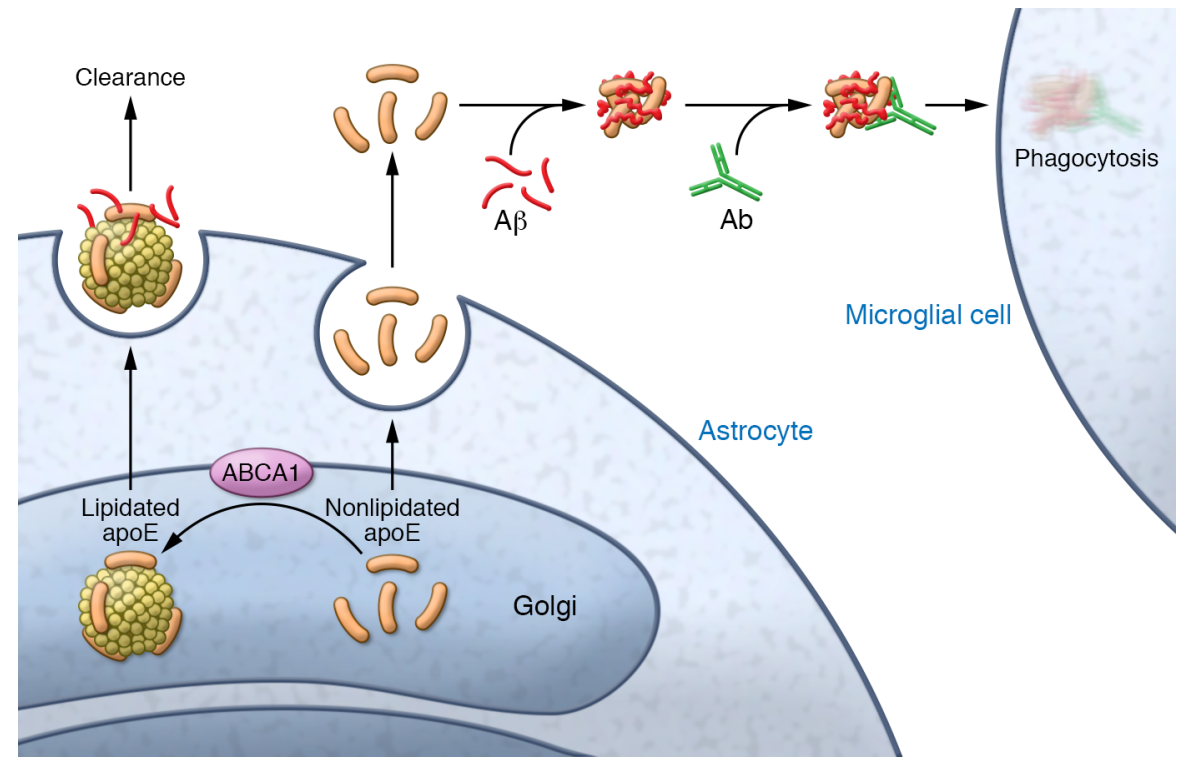

dated apoE may interfere with $\mathrm{A} \beta$ fibrillogenesis in vitro (19). However, studies in which ATP-binding cassette transporter A1 (ABCA1), a key cholesterol transporter in apoE lipidation, has been inactivated in mutant APP mice suggested that poorly lipidated murine apoE was associated with more severe $A \beta$ deposition (20). Moreover, the effect of ABCA1 deletion in APP/PS1/ $\mathrm{APOE} 4$ mice on $\mathrm{A} \beta$ deposition was more severe than the effect of $A B C A 1$ deletion in APP/PS1/APOE3 mice (21). If nonlipidated apoE 4 is an active accomplice in promoting $A \beta$ deposition, and if this isoform of apoE is indeed more prone to escape lipidation, then one might predict that transgenic overexpression of human APOE4 in mutant APP or APP/PS1 mice should dramatically worsen $\mathrm{A} \beta$ pathology. However, 2 separate studies using different transgenic approaches did not show that overexpression of human APOE4 exacerbated $\mathrm{A} \beta$ deposition $(22,23)$. Thus, although the evidence is clear that poor lipidation of apoE4 is associated with more severe $\mathrm{A} \beta$ deposition, it is still not entirely clear whether this effect is due to an active role of nonlipidated protein in promoting amyloidogenesis, or due to diminished abilities of poorly lipidated protein to participate in the clearance of $A \beta$ in the CNS (mechanisms reviewed in ref. 8).

\section{Summary and future directions} Whether nonlipidated apoE4 is an active or passive accomplice does not diminish the potential utility of targeting this par- ticular species of apoE by immune therapy if removing it also removes the initial assemblies of $A \beta$ that are the culprit in initiating $A \beta$ deposition. One advantage of targeting nonlipidated $\mathrm{CNS}$ apoE is that it appears to be a relatively minor fraction of the total systemic apoE burden. In this setting, passively administered antibody should survive longer in the plasma and potentially provide efficacy at a lower or less frequent dose. Indeed, data from the Liao study demonstrated that antibodies specific for nonlipidated apoE4 had longer plasma half-lives (9). These apoE antibodies also have the potential advantage of avoiding on-target side effects of $\mathrm{A} \beta$-targeting antibodies that produce amyloid-related imaging abnormalities. Moving this avenue of therapy forward will require a better understanding of the level of nonlipidated apoE4 in humans. It is noteworthy that a recent study identified the $A B C A 7$ gene, which encodes a cholesterol transporter expressed in CNS, as the strongest risk factor after APOE for early $A \beta$ pathology (24). The Liao study did not address whether treatments with apoE antibodies could clear preexisting $A \beta$ deposits - a key unknown in translating this therapy to patients who already have significant $A \beta$ pathology. Whether antibodies against nonlipidated apoE could displace $A \beta$-directed immune therapies as treatments to remove preexisiting $A \beta$ deposits is uncertain. The $A \beta$-directed immune therapies are poised for phase 3 clinical trials, and if they are the first to
Figure 1. Antibodies stimulate phagocytosis of apoE/A $\beta$ conglomerates to slow the formation of pathological amyloid deposits. Through the action of the ATP-binding cassette transporter A1 (ABCA1) most apoE acquires lipid in the Golgi and is secreted as proteolipid particles that bind monomeric $A \beta$ and facilitate clearance through multiple pathways. A small fraction of apoE, particularly apoE4, fails to assemble into proteolipid particles and is prone to bind assemblies of $A \beta$ that ultimately seed the formation of pathological $A \beta$ deposits. Antibodies that selectively bind the nonlipidated apoE opsonize the apoE/A $\beta$ conglomerates, leading to phagocytosis and degradation by resident microglia.

be approved in human therapy, then any new therapy would have to be evaluated in comparison to or in combination with the existing therapy. However, for the reasons outlined above, apoE antibodies targeting nonlipidated protein could ultimately emerge as the better choice for a preventive biotherapy in high-risk APOE4 carriers.

\section{Acknowledgments}

This work was supported by the National Institute on Aging (P50AG047266) and by the SantaFe HealthCare Alzheimer's Disease Research Center. I thank Benoit Giasson and Paramita Chakrabarty (University of Florida) for insightful and helpful discussions.

Address correspondence to: David R. Borchelt, Department of Neuroscience/ CTRND, Box 100159, 1275 Center Drive, University of Florida, Gainesville, Florida 32610, USA. Phone: 352.273.9664; Email: drb1@ufl.edu.

1. Corder EH, et al. Gene dose of apolipoprotein E type 4 allele and the risk of Alzheimer's disease in late onset families. Science. 1993;261(5123):921-923.

2. Strittmatter WJ, et al. Apolipoprotein E: highavidity binding to beta-amyloid and increased frequency of type 4 allele in late-onset familial Alzheimer disease. Proc Natl Acad Sci U S A. 1993;90(5):1977-1981.

3. Namba Y, Tomonaga M, Kawasaki H, Otomo E, Ikeda K. Apolipoprotein E immunoreactivity in cerebral amyloid deposits and neurofibrillary tangles in Alzheimer's disease and kuru plaque amyloid in Creutzfeldt-Jakob disease. Brain Res. 
1991;541(1):163-166.

4. Liu CC, Liu CC, Kanekiyo T, Xu H, Bu G. Apolipoprotein $\mathrm{E}$ and Alzheimer disease: risk, mechanisms and therapy. Nat Rev Neurol. 2013;9(2):106-118.

5. Breitner JC, et al. APOE-epsilon 4 count predicts age when prevalence of $\mathrm{AD}$ increases, then declines: the Cache County Study. Neurology. 1999;53(2):321-331.

6. Shi Y, et al. ApoE4 markedly exacerbates taumediated neurodegeneration in a mouse model of tauopathy. Nature. 2017;549(7673):523-527.

7. Mahoney-Sanchez L, Belaidi AA, Bush AI, Ayton S. The complex role of apolipoprotein $\mathrm{E}$ in Alzheimer's disease: an overview and update. JMol Neurosci. 2016;60(3):325-335.

8. Huynh TV, Davis AA, Ulrich JD, Holtzman DM. Apolipoprotein E and Alzheimer's disease: the influence of apolipoprotein $\mathrm{E}$ on amyloid- $\beta$ and other amyloidogenic proteins. J Lipid Res. 2017;58(5):824-836.

9. Liao F, et. al. Targeting of nonlipidated, aggregated apoE with antibodies inhibits amyloid accumulation. J Clin Invest. 2018;128(5):2144-2155.

10. Bales KR, et al. Lack of apolipoprotein E dramatically reduces amyloid beta-peptide deposition. Nat Genet. 1997;17(3):263-264.

11. Bales KR, et al. Human APOE isoformdependent effects on brain beta-amyloid levels in PDAPP transgenic mice. J Neurosci. 2009;29(21):6771-6779.

12. Youmans KL, et al. APOE4-specific changes in $\mathrm{A} \beta$ accumulation in a new transgenic mouse model of Alzheimer disease. J Biol Chem. 2012;287(50):41774-41786

13. Huynh TV, et al. Age-dependent effects of apoE reduction using antisense oligonucleotides in a model of $\beta$-amyloidosis. Neuron. 2017;96(5):1013-1023.e4

14. Liu CC, et al. ApoE4 accelerates early seeding of amyloid pathology. Neuron. 2017;96(5):1024-1032.e3.

15. Cerf E, Gustot A, Goo-rmaghtigh E, Ruysschaert JM, Raussens V. High ability of apolipoprotein E4 to stabilize amyloid- $\beta$ peptide oligomers, the pathological entities responsible for Alzheimer's disease. FASEB J. 2011;25(5):1585-1595.

16. Castano EM, et al. Fibrillogenesis in Alzheimer's disease of amyloid $\beta$ peptides and apolipoprotein E. Biochem J. 1995;306(pt 2):599-604.

17. Ma J, Yee A, Brewer HB, Das S, Potter H. Amyloid-associated proteins $\alpha 1$-antichymotrypsin and apolipoprotein E promote assembly of Alzheimer $\beta$-protein into filaments. Nature. 1994;372(6501):92-94.

18. Strittmatter WJ, et al. Binding of human apolipoprotein $\mathrm{E}$ to synthetic amyloid beta peptide: isoform-specific effects and implications for late-onset Alzheimer disease. Proc Natl Acad Sci US A. 1993;90(17):8098-8102.

19. Wood SJ, Chan W, Wetzel R. Seeding of $\mathrm{A} \beta$ fibril formation is inhibited by all three isotypes of apolipoprotein E. Biochemistry. 1996;35(38):12623-12628.

20. Wahrle SE, et al. Deletion of Abca1 increases $\mathrm{A} \beta$ deposition in the PDAPP transgenic mouse model of Alzheimer disease. J Biol Chem. 2005;280(52):43236-43242.

21. Fitz NF, et al. Abca1 deficiency affects Alzheimer's disease-like phenotype in human ApoE4 but not in ApoE3-targeted replacement mice. J Neurosci. 2012;32(38):13125-13136.

22. Lesuisse $\mathrm{C}$, et al. Hyper-expression of human apolipoprotein E4 in astroglia and neurons does not enhance amyloid deposition in transgenic mice. Hum Mol Genet. 2001;10(22):2525-2537.

23. Van Dooren T, et al. Neuronal or glial expression of human apolipoprotein e 4 affects parenchymal and vascular amyloid pathology differentially in different brain regions of double- and triple-transgenic mice. Am J Pathol. 2006;168(1):245-260.

24. Apostolova LG, et al. Associations of the Top 20 Alzheimer disease risk variants with brain amyloidosis [published online ahead of print January 16, 2018]. JAMA Neurol. https://doi. org/10.1001/jamaneurol.2017.4198. 